\title{
Performance, Operation and Detector Studies with the ATLAS Resistive Plate Chambers
}

\author{
Giulio Aielli ${ }^{a}$, Marcello Bindi ${ }^{b}$, Alessandro Polini ${ }^{c}$, \\ on behalf of the ATLAS Collaboration \\ ${ }^{a}$ INFN and University of Roma Tor Vergata \\ E-mail: aiellieroma2.infn.it \\ ${ }^{b}$ INFN Bologna, \\ E-mail: marcello.bindi@bo.infn.it \\ ${ }^{c}$ INFN Bologna, \\ E-mail: alessandro.polini@bo.infn.it
}

\begin{abstract}
Resistive Plate Chambers provide the barrel region of the ATLAS detector with an independent muon trigger and a two-coordinate measurement. The chambers, arranged in three concentric double layers, are operated in a strong magnetic toroidal field and cover a surface area of about $4000 \mathrm{~m}^{2}$. During 2011 the LHC has provided proton-proton collisions at $7 \mathrm{TeV}$ in the center-of-mass frame with a steady increase in instantaneous luminosity, summing up to about 5 $f b^{-1}$. The operational experience for this running period is presented along with studies of the detector performance as a function of luminosity, environmental conditions and working point settings. Non-event based information including in particular the large number of gas-gap currents, individually monitored with nA accuracy, have been used to study the detector behaviour with growing luminosity and beam currents. These data are shown to provide, when calibrated, an independent luminosity measurement and a crucial handle for understanding the ATLAS backgrounds well beyond of the scope of Muon triggering and detection. The measurements presented allow the planning of a strategy for the data taking in the next years, make some predictions about the performance with higher luminosities and improve the knowledge on RPC detector physics.
\end{abstract}

KEYWORDS: ATLAS; RPC; Muon; Luminosity; Background. 


\section{Contents}

1. Introduction 囵

2. The ATLAS Resistive Plate Chambers 2

3. Detector Status and Performance 3

4. Detector and DCS Operation

4.1 HV Working Point Correction 6

5. The Cavern Background measurement through RPC Gap-Currents 7

5.1 Offline Gap-Currents Analysis 9

5.2 Comparison with other Measurements in ATLAS

5.3 Data Monte Carlo Comparison 12

6. Luminosity and Detector Activation 13

6.1 Measurement Tecnique and Results

6.2 Comparison with ATLAS Measurements and Future Prospects 18

7. Conclusions 19

\section{Introduction}

In 2011 the bulk of the LHC program has provided proton-proton collisions at a center of mass energy of $7 \mathrm{TeV}$ with instantaneous luminosities up to $3.65 \times 10^{33} \mathrm{~cm}^{-2} \mathrm{~s}^{-1}$, totaling an integrated luminosity of $5.6 \mathrm{fb}^{-1}$. In addition the last month of the 2011 running also provided $166 \mu \mathrm{b}^{-1}$ of lead-lead nuclei collisions. This document presents the ATLAS Resistive Plate Chambers (RPCs) performance and operational data taking experience during this period, and studies done with data coming from the RPC detector control and monitoring system (DCS) during the operation with colliding beams. The main focus is on extracting fundamental knowledge form the present experience which will be useful to extrapolate the behavior at higher LHC luminosity from all the relevant points of view: detector and trigger performance, structural and ageing issues, service refurbishment, upgrade studies. In particular the introduction of the automatic correction of the working point with respect to the environmental conditions improved the detector performance as presented in Section B, where also timing and trigger performance are shown. The dynamic stabilization of 14 the working point with respect to the environmental conditions (Section 4 ) and the careful control 15 of the gas system also enabled to reliably use the detector current as background and luminosity 6 monitor as described in Sections 5 and 6 . The high granularity of the information has been also 


\section{The ATLAS Resistive Plate Chambers} $p_{T}$ triggers require hits also in the outer confirm layer.

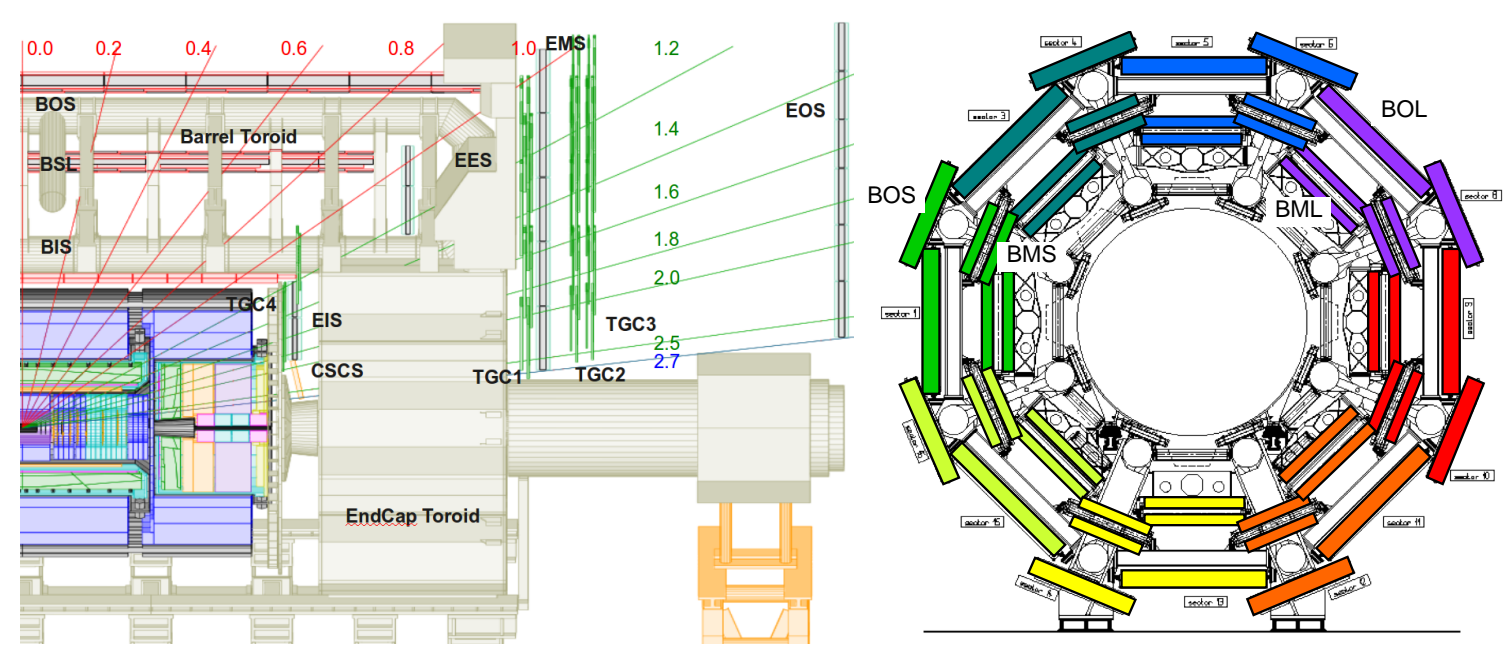

used to evaluate the cavern backgrounds distribution maps coming from the $p$ - $p$ interactions allowing a comparison with the available simulation and improve its description. The accurate and granular knowledge of the beam induced counting rate (through currents) and its linear relation with the LHC luminosity have several applications such as: pointing out shielding improvement, optimize the gas flow with respect to the background intensity, set up a precise monitoring system based on the ratios of local quantities to the instantaneous luminosity.

RPCs[1] provide the barrel region of the ATLAS detector [2] with an independent muon trigger and a two-coordinate measurement. The chambers (fig. 囵) are arranged in three concentric double layers (middle confirm, middle pivot, outer confirm) and are operated in a strong magnetic toroidal field covering a surface area of about $4000 \mathrm{~m}^{2}$. Each layer is organized in 16 sectors along the azimuthal coordinate $(\phi)$, to facilitate the overlap the even sectors are slightly smaller than the odd ones thus we refer respectively to large (L) and small (S) chambers. A low $p_{T}(<10 \mathrm{GeV})$ trigger requires a projective coincidence between hits in the pivot and the middle confirm layer while high

Figure 1. Views of the ATLAS muon system: $y-z$ plane (left) and $x-y$ plane (right). The 3 RPC double layers and the structure of small and large sectors is enhanced.

An ATLAS RPC is made of two layers, each with two $2 \mathrm{~mm}$ thick bakelite laminate plates and provides $2 \eta$ and $2 \phi$ strip planes. Made of phenolic and melaminic resins, the plates are kept apart at $2 \mathrm{~mm}$ by insulating spacers, enclosing a gas volume filled with a mixture of $\mathrm{C}_{2} \mathrm{H}_{2} \mathrm{~F}_{4}(94.7 \%)$ $\mathrm{C}_{4} \mathrm{H}_{10}(5 \%)-S F_{6}(0.3 \%)$. The external surface of the plates is coated with a thin layer of graphite paint to allow a uniform distribution of the high voltage along the plates. The smoothness of the inner surfaces is enhanced by means of a thin layer of linseed oil. The high voltage (HV) working point is chosen to be at $9.6 \mathrm{kV}$ at a temperature of $24^{\circ} \mathrm{C}$ and a pressure of 970 mbar. In these conditions the RPCs work in saturated avalanche mode inducing, for a minimum ionizing particle, a prompt charge of about $1 \mathrm{pC}$ on the pick-up strips and delivering in the gas an average total charge of $30 \mathrm{pC}$. The discharge electrons drift in the gas and the signal, induced on pick-up copper strips, 

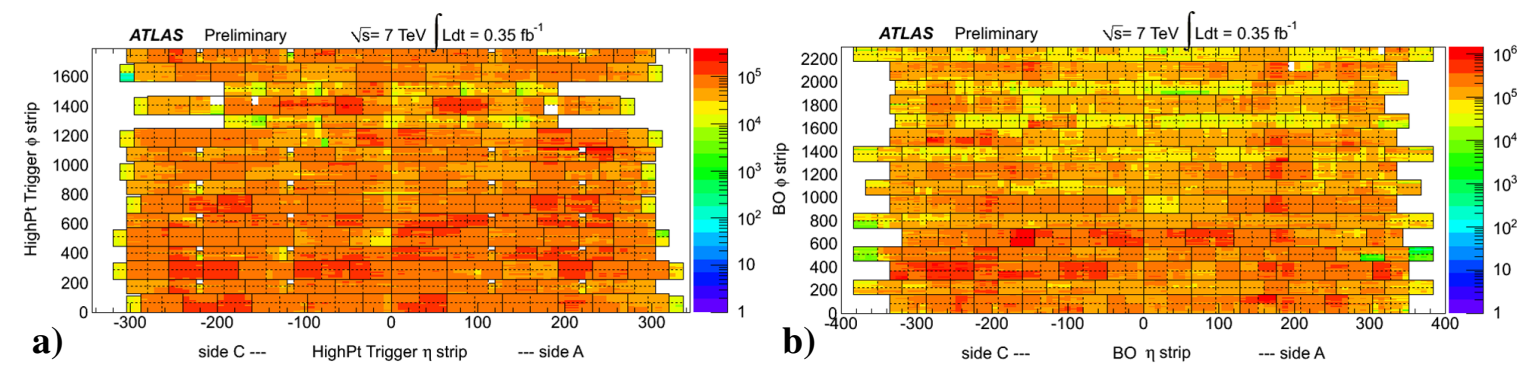

Figure 2. a) The spatial coincidence between $\eta$ and $\phi$ RPC pivot strips generating a High $p_{T}$ trigger threshold in terms of pseudo-rapidity and azimuthal angle coordinates, respectively. b) The spatial coincidence between $\eta$ and $\phi$ strips of the outer confirm double layer. Shown are data from the Muon_Physics stream taken between March and May 2011 when detector and beam flags were green.

is read out via capacitive coupling, and detected by the front-end electronics. Read-out strips have a typical width of $\sim 30 \mathrm{~mm}$ and are grouped in two $(\eta$ and $\phi)$ read-out panels with strips orthogonal to each other. Custom front-end electronics amplifies, discriminates and converts the detector signals to ECL standard. These signals are passed to the on-detector trigger electronics [3] which, by requiring appropriate coincidences in the $\eta$ and $\phi$ detector layers, provides ATLAS with a Level 1 trigger (L1) decision along with the detector data for accepted events.

\section{Detector Status and Performance}

The 2011 data taking has been very successful for the RPC system which has been running with a fraction of active readout channels of $97 \%$ (out of a total of $370 \mathrm{k}$ ) and an average active trigger area of $99 \sim 99.5 \%$ [ 困]. The number of gas volumes either disconnected or operated not at nominal conditions has been below $2 \%$ with 47 (out of 3592) being off due to broken gas inlets and 23 being kept at lower voltage for recovery due to bad gas supply or too high currents. The physics data taking has been stable with around $99 \%$ of the collected data flagged as good for physics analyses. Fig. 国 shows the detector coverage in strip units for the whole 2011 proton run. The plots reflect the detector chamber structure and coverage. The plots show a uniform coverage both for the trigger and the detector hits. The empty regions around $\phi$ strip 1400 and the 2 surrounding sectors come from the different design of the ATLAS detector in the lower sectors where the infrastructure to sustain the whole detector and the access paths for maintenance are located. Fig. Ba presents the efficiency per gas volume and compares the performance of 2011 with the one in 2010. As can be seen an important improvement was achieved in 2011 coming mainly from:

- the dynamic adjustment of the high voltage working point 7 as a function of the local environmental conditions (temperature and atmospheric pressure).

- a general detector consolidation

- precise timing calibration

On the working point adjustment more details are given in section 4 . The plot in Fig. $3 \mathrm{~b}$ shows the RPC timing performance. The profile is sharp and centered in the middle of the readout window indicating that the RPCs can be used as a precise timing system[5]. 

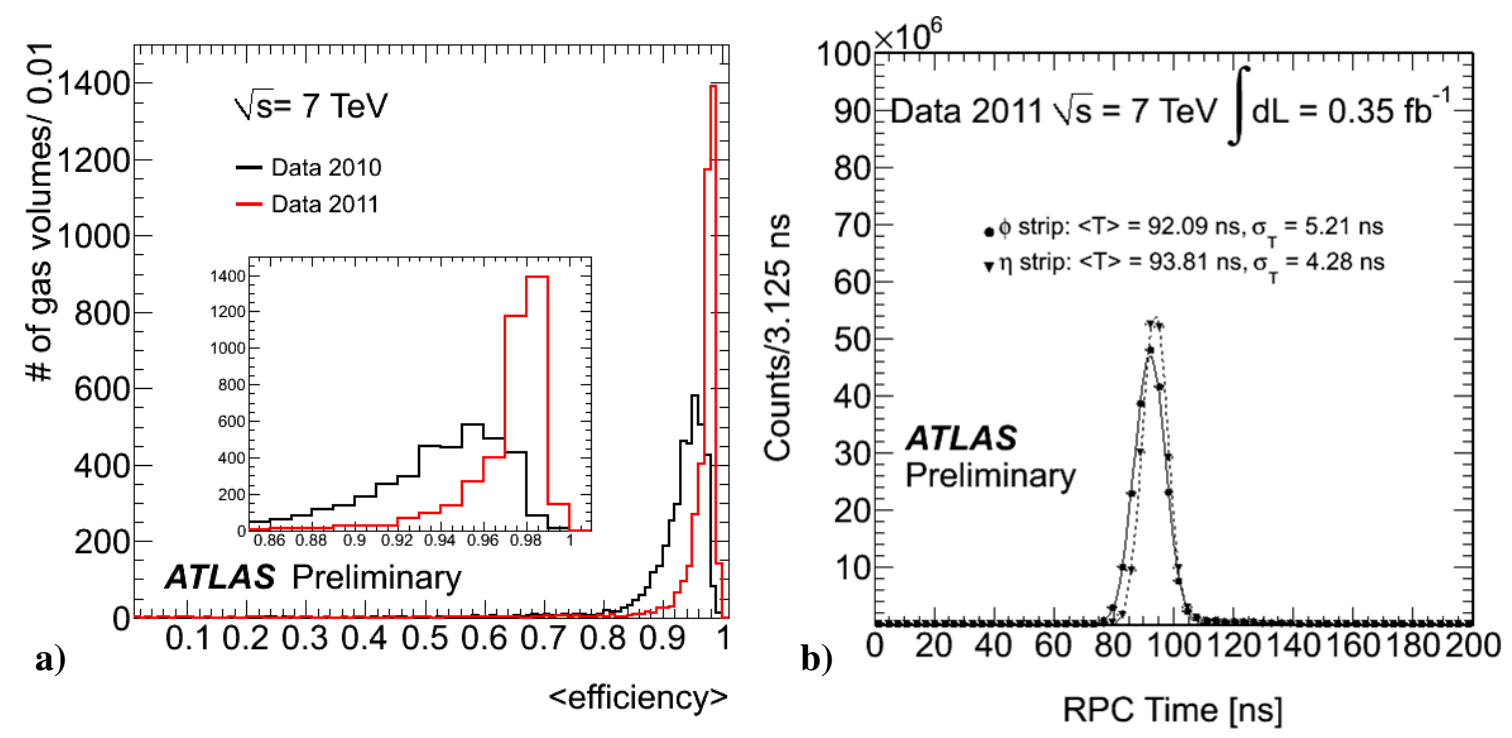

Figure 3. a) The distribution of the measured 2010 and 2011 RPC gas volume efficiencies defined by the positive response of at least one of the two views. The data correspond to all Muon Physics stream data for both years when detector and beam flags were green. b) The distribution of 2011 RPC time in readout hits for both views. No off-line time correction is applied and the time spread is dominated by the spread of signal propagation along the strip which can be corrected off-line. The data correspond to all Muon Physics stream data for 2011 when detector and beam flags were green

Fig. Ta shows the trigger efficiency as a function of the muon transverse momentum $p_{T}$. Six different trigger slots with increasing momentum threshold are displayed. The plateau efficiency is around $80 \%$ for low- $p_{T}$ triggers $(4,6,10 \mathrm{GeV})$; this value is expected because includes also the detector geometric acceptance. For the high- $p_{T}$ triggers $(11,15,20 \mathrm{GeV})$ the efficiency plateau is around $70 \%$ since a further coincidence in the outer plane is required, thus reducing the geometric acceptance.

The effect of the detector acceptance is clearly seen when plotting the efficiency as a function of $\phi$ as seen in Fig. \#b. The region with the 2 major dips corresponds to the lower ATLAS sectors where the support of the whole detector is located and where some chambers are missing to allow access inside the detector. In addition to this, the structure with small even and large odd sectors following the 8 large toroidal coils over $\phi$ can be also seen.

During 2011 the bulk of the proton collisions have been taken with 50 ns bunch spacing following the fact that the LHC was able to achieve higher luminosity and beam stability with respect to the running with $25 \mathrm{~ns}$ bunch spacing. Running with higher bunch spacing means that concurrently at equal luminosity the pileup $(\mu)$ increases and effects on detector efficiency, or linearity and saturation effects might come into the game.

Fig. 5 shows the RPC trigger performance as a function of the pileup $\mu$. Data corresponds to the period of highest luminosity with an average pileup $\langle\mu\rangle$ of 15 . No significant dependence on the RPC trigger efficiency is observed. 

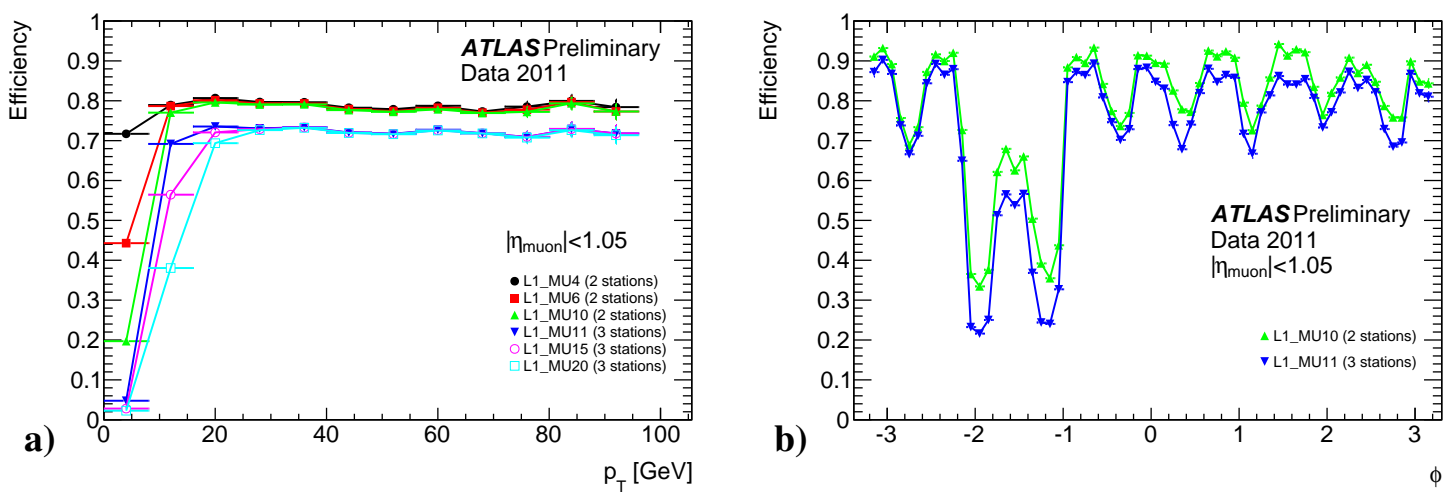

Figure 4. a) The L1 Muon Barrel trigger efficiency with respect to offline reconstructed combined muon (as a function of $p_{T}$ ), for the six nominal thresholds. The different acceptance between the low- $p_{T}$ thresholds (MU4, MU6, MU10) and the high- $p_{T}$ thresholds (MU11, MU15, MU20) is caused by the smaller coverage for the additional coincidence of the outer double layer. b) The L1 Muon Barrel trigger efficiency for the low- $p_{T}$ threshold MU10 and the high- $p_{T}$ threshold MU11 with respect to offline reconstructed combined muon, selected with $p_{T}>15 \mathrm{GeV}$, as a function of $\phi$. The efficiency has been determined with a tag and probe method using di-muon events. The data used correspond to total integrated luminosity of $380 \mathrm{pb}^{-1}$.
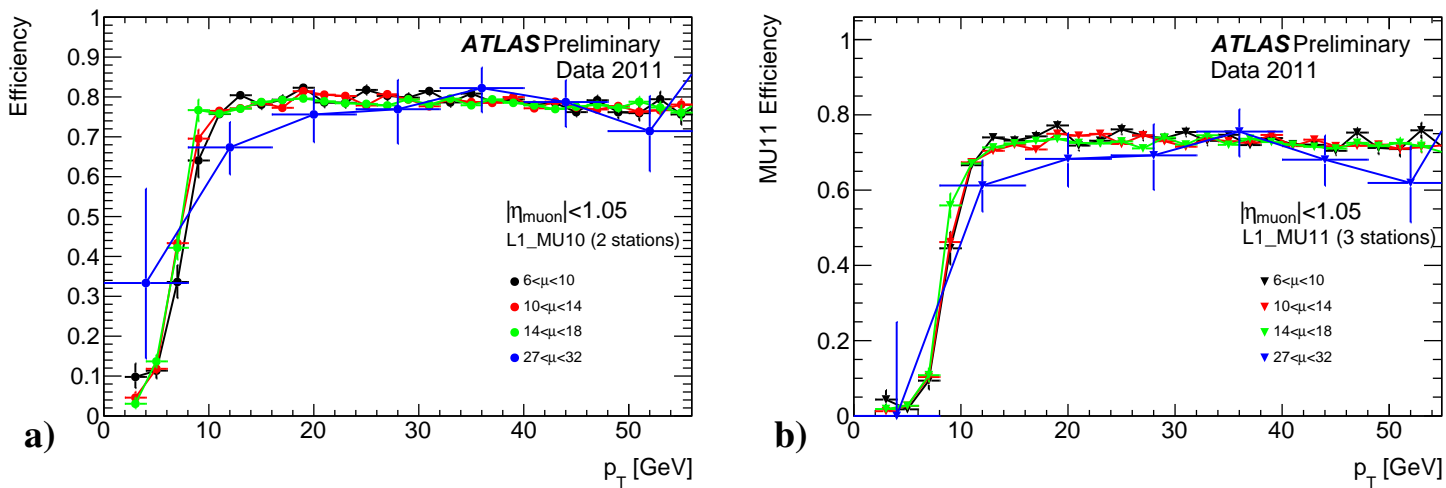

Figure 5. The L1 muon barrel trigger efficiency, for the 2-station threshold MU10 (a) and for the 3-station threshold MU11 (b), with respect to offline reconstructed combined muon as a function of the muon $p_{T}$. The efficiency curves are shown for different ranges of the average number of interactions per bunch crossing, $\mu$. The efficiency has been determined with a tag and probe method using di-muon events. The data used correspond to total integrated luminosity of $380 \mathrm{pb}^{-1}$.

\section{Detector and DCS Operation}

The RPCs benefited in 2011 run from the introduction of several automatic monitoring and control tools to simplify the detector operation and optimize the data taking conditions. The number of shifters was reduced to a single person taking care of the whole ATLAS muon system which includes also trigger chambers in the endcap region and precision chambers.

One further improvement was to add to the RPC DCS the full automatic control of the HV settings. These are automatically adjusted

- to follow the different LHC beam phases from injection, stable beams, to the final dump 
- to compensate for the local changes of the environmental conditions (mainly local temperature and atmospheric pressure)

- to automatically check the individual gas-gaps currents, recalibrate at each end of fill the pedestals

- provide an online measurement of the cavern background rates and an instantaneous luminosity

The DCS is in charge of safely operating and monitoring the detector power system including the detector HV and LV supply. In the RPC DCS [6], a large number of settings (DAC 4000) and monitoring (ADC 6500) channels has been integrated into the system to optimize the detector performance and allow a fine monitoring at the level of the single individual gas-gap $(\sim 3600)$. The remaining ADC channels are used to monitor with high granularity the current draw of the front end electronics and RPC gas and environmental sensors (temperature, atmospheric pressure, relative humidity and gas flow). The ability to control by tuning thresholds, and monitoring the current of each RPC gap has shown to be very powerful for the detector operation both for tracing problems and fine tune the detector. This is particularly important as the RPC performance and aging is strongly related to the environmental parameters, namely the temperature $(T)$, the atmospheric pressure $(P)$, and the relative humidity.

\subsection{HV Working Point Correction}

The gas gain, the noise rate and the dark current of the chamber depend on these parameters following the formula:

$$
V_{\text {appl }}=V_{\text {eff }} \cdot\left(T_{0} / T\right) \cdot\left(P / P_{0}\right)=\rho \cdot V_{\text {eff }}
$$

where $V_{\text {appl }}$ is the applied voltage, $T, P$ are the environment measurements and $T_{0}, P_{0}, V_{\text {eff }}$ are the reference environmental values and HV settings. The data of about 300 temperature sensors located on the chambers across the whole system (along with the pressure measurement) are used to adjust the round $280 \mathrm{HV}$ channels. In order to avoid wrong or over-corrected HV set-points, the data from the adjacent sensors is used only if within a range of validity and then combined. Following the limited granularities of the HV channels and of the temperature sensors compared to the number of gas-gaps $(\sim 3600)$ only a coarse correction averaged over a larger volume can be achieved. Conservative settings, which were confirmed from previous studies during detector tests [7] and from data with beam in 2009-2010 [8] were used: a factor of 0.8 for the atmospheric pressure term and of 0.5 to the temperature. Furthermore, for detector safety, a limit to the HV $(9500 \mathrm{~V})$ for chambers with temperature above $26^{\circ} \mathrm{C}$ was set along with independent procedures steadily monitoring the gas flow and the gas-gap currents which would lower the corresponding HV set point in case of abnormal readout values. During nominal running, the environmental correction updates every couple of minutes refreshing the setpoints of the HV channels which are kept with the lowest ramp up/down speed. The correction is automatically disabled for the periods of no beam or during run transition. An example of the HV working point correction as a function of time is shown in Fig. 6. From the beginning of 2011, the correction described has been stably used allowing a general improvement in the detector performance and efficiency.

The size and high granularity of the information read out and archived by the DCS is a valuable source of data for detector physics. This information correlated with the detector readout and layer 

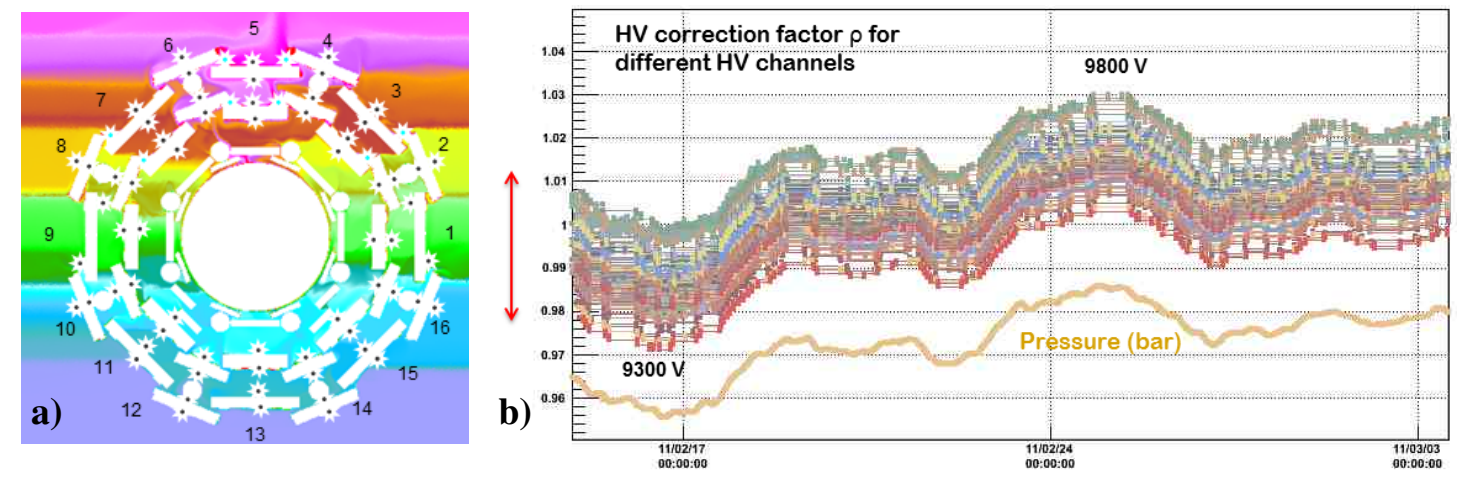

Figure 6. a) Artistic view of the temperature distribution on the RPC system. b) An example of the environmental correction to the RPC HV working point. The correction factor $\rho$ applied to the nominal voltage for a bunch of $\mathrm{HV}$ channels is shown as a function of time. Chambers belonging to different detector areas being at different temperatures show a spread in the applied voltage of up to $\pm 150 \mathrm{~V}$. In normal operation the temperature differences remain constant while continuous adjustment follows the trend of atmospheric pressure moving the operating voltage by up to the $3 \%$ of the nominal voltage.

redundancies has allowed to monitor and intervene in case of problems as for instance in case of chambers with high current or insufficient gas flow. Furthermore the gas-gap currents of the RPCs, measured by the DCS with a sensitivity of $2 \mathrm{nA}$, allow for a precise estimation of background and beam effects. The monitored currents and pedestals, the environmental variables and the beam information are used to estimate the average radiation induced counting rate per surface unit to study beam background and activation effects and their relation with the integrated and instantaneous luminosity, as demonstrated in sections 5 and 6 . Part of this features are implemented online by the DCS allowing instantaneous monitoring and publishing of the background distribution. Fig. 7 shows the online distributions over the longitudinal $(z)$ and azimuthal $(\phi)$ coordinates of the beam induced detector currents as measured and normalized by the detector surface for the 3 double layers of RPC chambers.

These measurements and the studies presented in the next sections, have suggested to adjust the gas flow to the individual chambers in the shutdown after the 2011 run. A new scheme with individually tuned impedances as function of the chamber volume and the expected integrated radiation was installed across the whole detector[9].

\section{The Cavern Background measurement through RPC Gap-Currents}

The detection of a ionizing particle is associated to the production of an electron avalanche in the gas. The total charge delivered has to be compensated by the power supply with a time constant of about $20 \mathrm{~ms}$ driven by the total capacitance and resistance of the system [10]. The average value of the detector current, cleaned up from all detector systematic contributions, represents the average charge per second delivered due to the incident particle rate. By knowing the average charge per particle delivered in the gas $\left\langle Q_{t o t}\right\rangle$, the gap current $I_{\text {gap }}$ and its area $A_{\text {gap }}$, the average particle rate $\mathrm{R}$ incicent on each gap can be extracted as:

$$
R=\frac{I_{\text {gap }}}{\left\langle Q_{\text {tot }}\right\rangle \cdot A_{\text {gap }}} .
$$




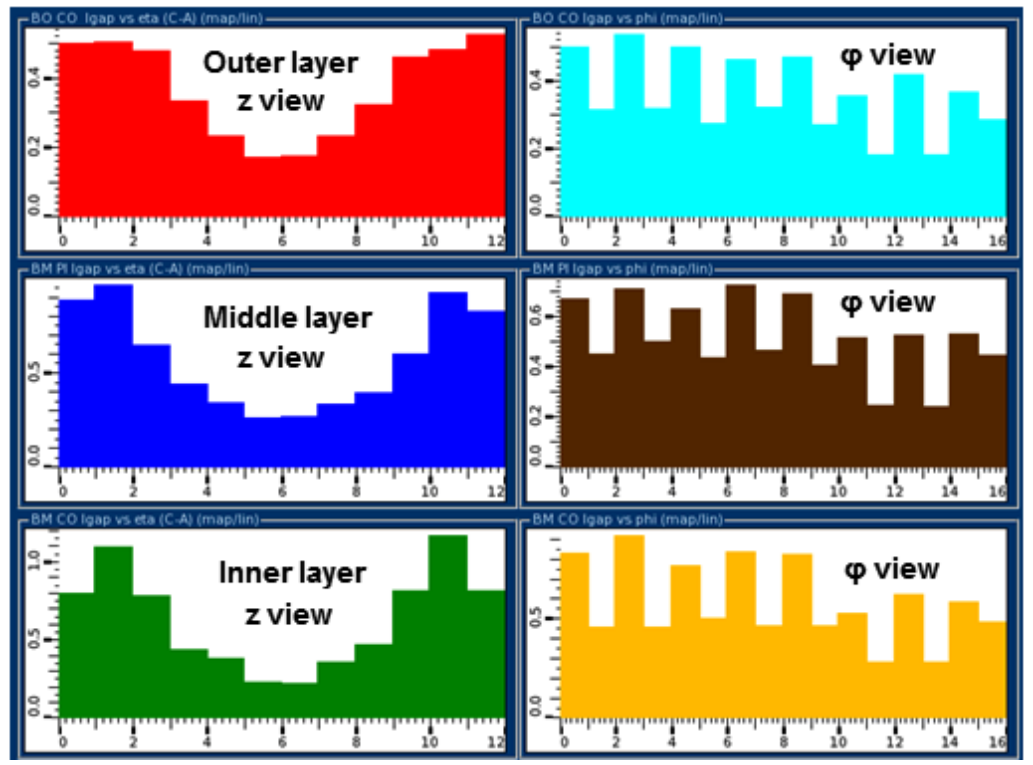

Figure 7. DCS online plots displaying the pedestal subtracted gas-gap currents (per unit area) for the 3 double layers of RPC chambers. The views in $z$ and $\phi$ are shown.

The sensitivity is driven by the precision of the volt-amperometric method used to measure the detector current flowing on a $100 \mathrm{k} \Omega$ resistor in parallel to a $10 \mathrm{nF}$ capacitor, placed in series on the HV return wire before joining with the common detector ground. The voltage drop across the resistor is read with CAEN A-3801, a 128 channels ADC module having a sensitivity of 0.2 $\mathrm{mV}$ which corresponds to $2 \mathrm{nA}$, equivalent to about $30 \mathrm{~Hz} / \mathrm{m}^{2}$ of particle rate. The instrument can average a programmable number of $1 \mathrm{~ms}$ samples up to 250, within a readout cycle of about 1s. Thus the measurement duty cycle is very high if compared to the hit counting based method of about $4 \times 10^{-5}$ given by the readout window ( $2 \mu \mathrm{s}$ for the MDTs and 200ns for the RPCs) multiplied by the data acquisition rate of the order of $200 \mathrm{~Hz}$. By this means the time necessary to collect an equivalent sample is about 6000 times faster, allowing in principle sensitive measurement at very low luminosity ( $\mathscr{L}=6 \times 10^{28} \mathrm{~cm}^{-2} \mathrm{~s}^{-1}$ is the lowest measured signal). To enhance the statistics the DCS acquires and averages several current measurements, recording the resulting moving average about every minute. Moreover the working point correction performed by the DCS removes the environmental effects as explained above.

The gas-gaps installed in the ATLAS barrel region are organized in doublets of identical gaps. A chamber can contain up to 4 doublets coupled side by side in $\phi$ or in $z$ direction, or both. The statistical combination of all the gaps provides the best possible estimate of the average background rate in the RPC area. A conversion factor of $30 \mathrm{pC} /$ count at the standard working point of $9600 \mathrm{~V}$ ( $\mathrm{P}=975 \mathrm{mbar}$ and $\mathrm{T}=24^{\circ} \mathrm{C}$ has been estimated for photons at the CERN GIF (Gamma Irradiation Facility) with a ${ }^{137} \mathrm{Cs}$ source [11] and with a ${ }^{60} \mathrm{Co}$ source in laboratory tests [12]. Other cases such as neutrons, are neglected in this analysis. This approximation is justified by the fact that on one hand in the barrel the neutron hits are negligible with respect to the photons due to the very low sensitivity [13] while on the other hand a highly saturated regime should strongly suppress the signal charge dynamical range [14]. 


\subsection{Offline Gap-Currents Analysis}

The raw rata from the RPC currents are reprocessed off-line to improve the quality of the measurement cleaning up the noisy channels and improving the statistical error. To cleanup the noise the facing gaps of the same doublet (the minimal RPC subset measuring the background in 3D) are compared. Having the same surface and position, these are very correlated and expected to measure the same background intensity. The average RPC working current without beam is of the order of $100 \mathrm{nA} \mathrm{m}^{-2}$ and is subtracted using the calibration data after each beam dump. The best value for the doublet is determined looking at Fig.8. If the difference of the two currents is less than $20 \%$ the average current will be taken as representative of the doublet; otherwise the lowest of the two is taken. Other checks are applied to avoid fake values due to disconnected gaps or readout failures or known noisy cases. The rate in $\mathrm{Hz} / \mathrm{cm}^{2}$ is obtained by using $30 \mathrm{pC} /$ count as explained above.

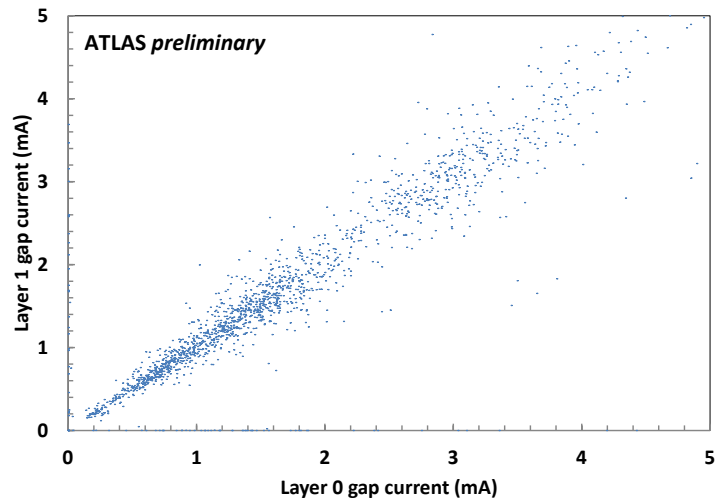

Figure 8. Scatter plot of measured current of homologous gas-gaps occupying the same position in the space while belonging to different layers.

The map shown in Fig. 9 represents the rate values as a function of doublet position within the ATLAS barrel in cylindrical coordinates. The radius corresponds to the chamber layer distance from the beam axis (e.g. middle and outer layer stations); the longitudinal coordinate $z$ is given by the RPC unit identifier along the beam axis (values are given relatively to the interaction point considering the clockwise circulating beam, named beam 1); the azimuthal coordinate $\phi$ is given by the sector and semi sector identifiers running clockwise around beam 1. These maps show counting rate in $\mathrm{Hz} / \mathrm{cm}^{2}$ in each bin, the color scale, given to help the plot understanding is generated by a series of proportional shades of color normalized to the maximum and minimum rate values. The $z$ and $\phi$ projective averages are also provided, as well as the global average at the bottom right corner of each table. In general we observe that the rate tend to increase at higher $z$ for the same radius as expected due to the higher value of $\eta$; moreover the systematic rate difference between odd and even sectors due to the effect of the toroid coils shadowing the even sectors and acting as absorber material. Going more in details two hot regions are visible, in particular in the innermost chamber layer for chamber id along $z= \pm 5$. This is due to the known unshielded crack present until 2011 between the calorimeter and the small wheel shielding, called J-disk as described in [17]. For the 2012 runs the shielding has been improved to suppress the rate excess. The lower ATLAS sectors $(12,13$ and 14) show a reduced counting rate due to the presence of the ATLAS feet steel supports. 


\begin{tabular}{|c|c|c|c|c|c|c|c|c|c|c|c|c|c|c|c|c|c|c|c|c|c|c|c|}
\hline \multirow{2}{*}{$\begin{array}{l}\text { Sector } \\
\Phi \text { ld. }\end{array}$} & \multicolumn{22}{|c|}{ RPC unit Id. along Z direction } & \multirow[t]{2}{*}{ 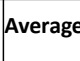 } \\
\hline & -7 & -6.2 & -6.1 & -5 & -4 & -3.2 & -3.1 & -2.2 & -2.1 & -1.2 & -1.1 & 1.1 & 1.2 & 2.1 & 2.2 & 3.1 & 3.2 & 4 & 5 & 6.1 & 6.2 & 7 & \\
\hline 1.1 & & 28 & 26 & 36 & 25 & 14 & 10 & 12 & 9 & & 8 & 5 & 5 & 9 & 10 & 10 & 14 & 19 & 37 & 26 & 24 & 22 & 18 \\
\hline 1.2 & 22 & 25 & 25 & 34 & 23 & 16 & 11 & 12 & 11 & & 7 & 6 & 6 & 9 & 11 & 11 & 14 & 21 & 38 & 25 & 26 & 22 & 18 \\
\hline 2 & & 11 & 18 & 18 & 14 & 10 & 8 & 7 & 7 & 5 & 5 & 4 & 5 & 6 & 7 & 9 & 9 & 15 & 19 & 17 & 15 & & 11 \\
\hline 3.1 & & 26 & 26 & 36 & 22 & 14 & 12 & 11 & 10 & 6 & 7 & 7 & 7 & 11 & 11 & 11 & 13 & 20 & 33 & 25 & 26 & & 17 \\
\hline 3.2 & & 26 & 31 & 32 & 21 & 14 & 11 & 13 & 11 & 8 & 7 & 7 & 8 & 11 & 12 & 14 & 14 & 20 & 32 & 24 & 25 & & 18 \\
\hline$\dddot{4}$ & & 9 & 16 & 17 & 15 & 10 & 8 & 7 & 7 & 5 & 5 & 5 & 6 & 7 & 7 & 8 & 10 & 16 & 17 & 17 & 16 & & 11 \\
\hline 5.1 & 2 & 22 & 22 & 29 & 17 & 13 & 10 & 12 & 10 & 7 & 6 & 6 & 7 & 12 & 14 & 11 & 11 & 16 & 26 & 21 & 21 & & 16 \\
\hline 5.2 & 22 & 23 & 22 & 26 & 18 & 14 & 11 & 17 & 11 & 7 & 6 & 6 & 7 & 9 & 11 & 9 & 12 & 17 & 24 & 23 & 22 & 22 & 15 \\
\hline 6 & & 11 & 17 & 18 & 14 & 9 & 8 & 7 & 8 & 6 & 5 & 5 & 6 & 7 & 7 & 9 & 10 & 17 & 18 & 19 & 16 & & 11 \\
\hline 7.1 & & 26 & 25 & 27 & 19 & 15 & 11 & 11 & 10 & & 7 & 7 & & 11 & 13 & 11 & 14 & 18 & 28 & 25 & 23 & 26 & 17 \\
\hline 7.2 & 22 & 27 & 23 & 25 & 20 & 13 & 10 & 11 & 9 & & 7 & 7 & & 9 & 11 & 11 & 15 & 19 & 31 & 25 & 24 & 26 & 17 \\
\hline 8 & & 10 & 17 & 17 & 14 & 9 & 8 & 7 & 6 & 5 & 5 & 5 & 5 & 6 & 7 & 8 & 10 & 16 & 17 & 17 & 15 & & 10 \\
\hline 9.1 & & 27 & 23 & 35 & 21 & 15 & 11 & 12 & 10 & & 6 & 6 & & 9 & 11 & 12 & 13 & 19 & 32 & 24 & 25 & & 19 \\
\hline 9.2 & 22 & 27 & 23 & 34 & 21 & 14 & 12 & 9 & 9 & & 5 & 7 & & 9 & 9 & 9 & 13 & 19 & 33 & 24 & 25 & 22 & 17 \\
\hline 10 & & 11 & 17 & 18 & 14 & 9 & 7 & 6 & 6 & 4 & 4 & 3 & 5 & 6 & 6 & 7 & 8 & 13 & 17 & 17 & 16 & & 10 \\
\hline 1i.i & & 23 & 21 & 25 & 15 & 10 & 8 & 8 & 7 & & 4 & 4 & & 7 & 7 & 8 & 9 & 14 & 25 & 20 & 22 & 14 & 13 \\
\hline 11.2 & 22 & 16 & 15 & 14 & 10 & 7 & 6 & 6 & 5 & & 3 & 3 & & 5 & 5 & 6 & 7 & 9 & 14 & 14 & 15 & 14 & 9 \\
\hline 12 & & & & & & 21 & 7 & 7 & 6 & 4 & 3 & 3 & 4 & 6 & 7 & 11 & 9 & & & & & & 6 \\
\hline 13.i & & 25 & 24 & 24 & & 12 & 9 & 8 & 7 & 7 & 5 & 4 & 5 & 7 & 9 & 8 & 11 & & 25 & 24 & 26 & 23 & 14 \\
\hline 13.2 & 22 & 25 & 23 & 22 & & 10 & 10 & 9 & 7 & 7 & 5 & 4 & 5 & 8 & 9 & 9 & 11 & & 26 & 21 & 24 & 23 & 13 \\
\hline 14 & & & & & & & 11 & 7 & 5 & 4 & 3 & 3 & 4 & 6 & 7 & 12 & 10 & & & & & & 6 \\
\hline 15.1 & 23 & 24 & 11 & 14 & 10 & 8 & 7 & 6 & 5 & & 3 & 3 & & 5 & 5 & 7 & 9 & 10 & 14 & 15 & 15 & & 9 \\
\hline 15.2 & 23 & 16 & 14 & 27 & 16 & 11 & 9 & 9 & 7 & & 4 & 4 & & 6 & 9 & 10 & 11 & 15 & 28 & 21 & 22 & 22 & 14 \\
\hline 16 & & 11 & 18 & 19 & 14 & 9 & 8 & 6 & 6 & 5 & 5 & 4 & 4 & 6 & 6 & 7 & 9 & 16 & 18 & 18 & 14 & & 10 \\
\hline Average & 24 & 18 & 20 & 24 & 17 & 12 & 9 & 9 & 8 & 5 & 5 & 5 & 5 & 7 & 8 & 10 & 11 & 16 & 24 & 20 & 20 & 24 & 13 \\
\hline
\end{tabular}

Figure 9. Average background rate distribution map for the RPC middle for $\mathscr{L}=10^{34} \mathrm{~cm}^{-2} \mathrm{~s}^{-1}$. The rate in $\mathrm{Hz} / \mathrm{cm}^{2}$ is shown for each individual gap position identified by its longitudinal and azimuthal position

\subsection{Comparison with other Measurements in ATLAS}

Cavern background refers to the diffuse background from $p$ - $p$ collisions filling the ATLAS detector cavern. It mostly consists of neutral, low energy and long lived particles. Figure 10 shows the expected background abundance in different areas of the ATLAS muon spectrometer, by particle type and energy. This prediction has been obtained by a FLUKA based simulation for $7 \mathrm{TeV}+7$ $\mathrm{TeV} p$ - $p$ collisions. The two most abundant particle types are photons and neutrons.

The cavern background study is necessary to quantify the background levels, how these might evolve as a function of the LHC luminosity and beam energies and to verify the Monte Carlo prediction done before the LHC start with very large uncertainties. This is particularly crucial to check the present detector reliability and to foresee in advance the upgrades for detectors and shielding having the appropriate performance. Fig. [1 shows a view of the ATLAS detector with particular focus on the Muon Detectors which include, besides the RPC spectrometer, also Monitored Drift Tubes (MDT) over the whole rapidity coverage, and Cathode Strip Chambers and Thin Gap Chambers in the forward region. A combined measurement using data from the Muon system is in preparation [17]].

The cavern background is actually measured by all the muon detectors to guarantee the maximum possible coverage in terms of detector volume and to comparatively study the different detector technology behavior to the background exposure, in terms of sensitivity and measurement systematic errors. Moreover the cavern background has been simulated with FLUGG, providing the expected rates relative to each detector type and region. In the barrel region $(|\eta|<1.05)$ the comparison is ensured by the MDTs and RPCs wich are bundled in to muon stations everywhere (except in the innermost region), thus they can be easily compared for the background measurement. 


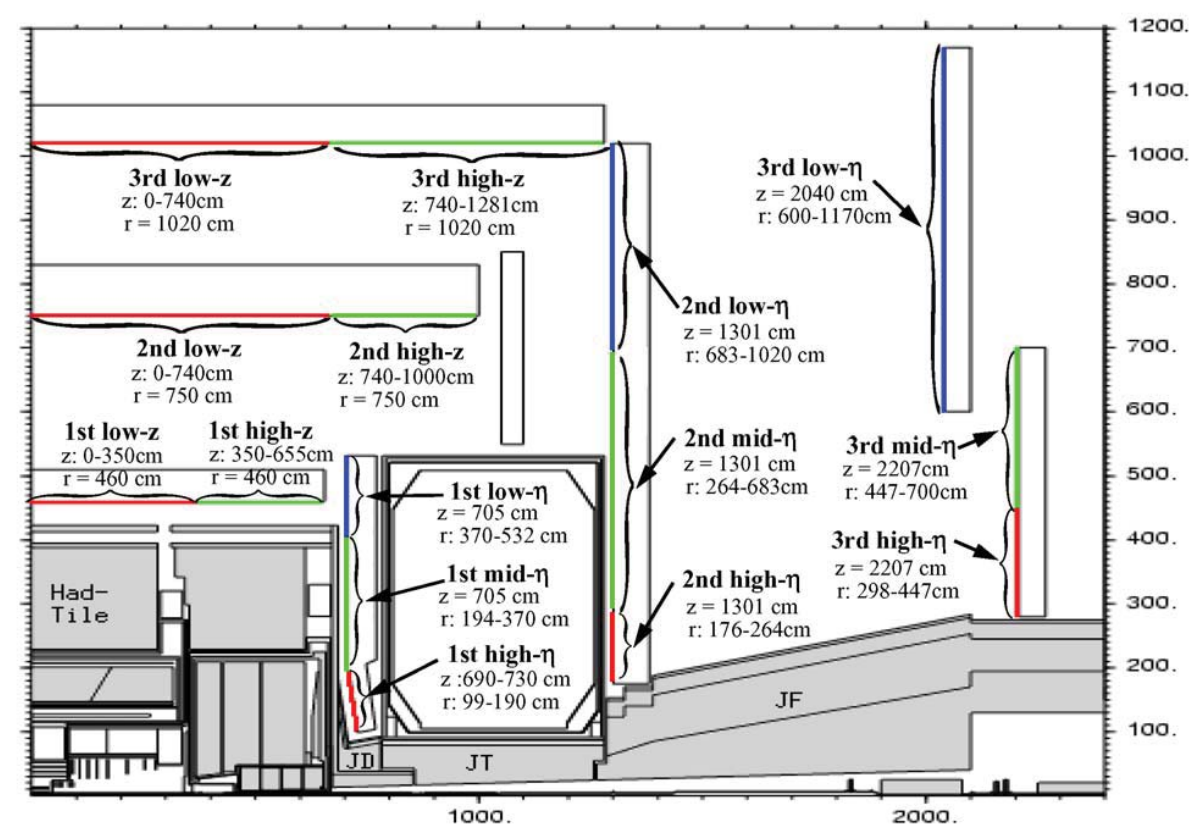

\begin{tabular}{||l||c|c|c|c||c|c|c|c|}
\hline \multicolumn{1}{|c||}{} & \multicolumn{3}{c||}{ Fluences $\left(\mathrm{kHz} / \mathrm{cm}^{2}\right)$} & \multicolumn{3}{c|}{ Currents $\left(\mathrm{Hz} / \mathrm{cm}^{2}\right)$} \\
\cline { 2 - 8 } Position & \multicolumn{3}{c||}{$\begin{array}{c}\text { Neutrons } \\
\text { Total }\end{array}$} & $\begin{array}{c}\text { Photons } \\
>\text { 30keV }\end{array}$ & $\begin{array}{c}\text { Ch.hads } \\
>10 \mathrm{MeV}\end{array}$ & $\begin{array}{c}\text { Protons } \\
>10 \mathrm{MeV}\end{array}$ & $\begin{array}{c}\mu^{+-} \\
>10 \mathrm{MeV}\end{array}$ & $\begin{array}{c}\mathrm{e}^{+-} \\
>0.5 \mathrm{MeV}\end{array}$ \\
\hline \multicolumn{1}{|c||}{} & & & & & & & & \\
Barrel & & & & & & & & \\
1st,low-z & 5.08 & 1.47 & 0.10 & 1.81 & 2.79 & 1.89 & 1.89 & 9.30 \\
1st,high-z & 5.25 & 1.47 & 0.07 & 1.88 & 2.24 & 1.86 & 2.55 & 13.29 \\
2nd,low-z & 4.97 & 1.20 & 0.10 & 2.19 & 0.69 & 0.64 & 0.37 & 7.00 \\
2nd,high-z & 6.28 & 1.72 & 0.30 & 4.14 & 1.88 & 1.81 & 0.77 & 11.81 \\
3rd,low-z & 4.45 & 0.86 & 0.08 & 1.90 & 0.32 & 0.31 & 0.09 & 5.96 \\
3rd,high-z & 4.87 & 1.02 & 0.18 & 2.71 & 0.98 & 0.97 & 0.39 & 8.10 \\
Forward & & & & & & & & \\
1st,low-eta & 14.23 & 4.80 & 0.66 & 4.93 & 4.89 & 4.38 & 3.60 & 33.57 \\
1st,mid-eta & 39.33 & 14.38 & 1.63 & 11.64 & 9.83 & 8.58 & 15.03 & 64.30 \\
1st,high-eta & 81.72 & 34.23 & 5.72 & 17.51 & 40.46 & 35.10 & 91.38 & 162.67 \\
2nd,low-eta & 5.55 & 1.51 & 0.25 & 3.18 & 1.98 & 1.93 & 0.25 & 11.91 \\
2nd,mid-eta & 8.36 & 2.61 & 0.49 & 4.43 & 6.45 & 6.20 & 1.20 & 23.58 \\
2nd,high-eta & 16.70 & 5.83 & 1.23 & 8.11 & 19.55 & 17.63 & 4.05 & 57.90 \\
3rd,low-eta & 3.16 & 0.48 & 0.06 & 1.40 & 1.53 & 1.47 & 0.27 & 7.41 \\
3rd,mid-eta & 3.09 & 0.47 & 0.05 & 1.31 & 1.25 & 1.21 & 0.32 & 6.41 \\
3rd,high-eta & 2.95 & 0.48 & 0.05 & 1.21 & 1.06 & 0.97 & 0.46 & 7.32 \\
& & & & & & & & \\
\hline
\end{tabular}

Figure 10. Typical distribution of background by particle type and energy in the muon spectrometer region predicted in a FLUKA-based simulation.

The barrel is characterized by a lower background intensity with respect to the forward region (endcap), largely dominated by photon hits, and negligible beam halo contamination, thus allowing very clean measurements. The endcap region $(1.05<\eta<2.4)$ is covered by TGCs and MDT chambers installed on separated layers at different distances from the interaction point. In the innermost region and closest to the beam axis $1.9<\eta<2.7$ the CSC is the only muon detector installed, thus crucial to the background study in the most critical area. 
Two principal measurement strategies have been pursued, complementary to each other, based respectively on hits collected through the DAQ and detector currents collected through DCS. In the first case hits from all the muon chambers are read out via the event data acquisition path. To minimize the bias from the trigger the data come from minimum bias or random filled trigger stream. The result is sensitive to the pileup effect due to the bunch structure of the beam and to the long tail of the time spectrum of the hits correlated to the bunch crossing. The HV current measurements integrate the signal over a period much longer than the LHC revolution frequency therefore are insensitive to the bunch structure and to the trigger. Their spatial granularity is generally poorer than that from hits which is driven from the chamber granularity. In general the charge signal amplitude depends on the primary ionization which depends on the type of particles. This uncertainty has to be taken in to account through appropriate conversion factors.

The table 1 summarizes the principal feature of the different methods and detectors potentiality.

\begin{tabular}{|c|c|c|c|c|c|c|c|c|}
\hline Type & $\begin{array}{l}\text { Systematic } \\
\text { error }\end{array}$ & Coverage & $\begin{array}{l}\text { Sensitivity } \\
\mathscr{L}\left[\mathrm{cm}^{-2} \mathrm{~s}^{-1}\right]\end{array}$ & $\begin{array}{l}\text { Granularity } \\
\text { (spacial) }\end{array}$ & $\begin{array}{c}\Delta \mathrm{t} \\
\min \end{array}$ & $\begin{array}{l}\text { Particle } \\
\text { Id }\end{array}$ & Timing & $\int$ time \\
\hline $\begin{array}{l}\text { RPC } \\
\text { current }\end{array}$ & $\begin{array}{c}\text { conversion factor } \\
\mathrm{P}, \mathrm{T}, \mathrm{RH}\end{array}$ & $\eta<1.05$ & $6 \times 10^{28}$ & $\begin{array}{l}3600 \text { gaps } \\
\text { (barrel) }\end{array}$ & $30 \mathrm{~s}$ & n.a. & n.a. & $5 \%$ \\
\hline $\begin{array}{l}\text { RPC } \\
\text { hits }\end{array}$ & $\begin{array}{l}\text { Efficiency } \\
\text { strip noise }\end{array}$ & $\eta<1.05$ & $10^{31}$ & $\begin{array}{c}4 \times 10^{5} \\
2 \mathrm{D} \text { strips }\end{array}$ & $\sim$ hour & $\begin{array}{c}\mathrm{n}, \gamma \\
\text { charged }\end{array}$ & $<3 \mathrm{~ns}$ & $\begin{array}{c}200 \\
\mathrm{~ns} / \mathrm{evt}\end{array}$ \\
\hline $\begin{array}{l}\text { MDT } \\
\text { current }\end{array}$ & $\begin{array}{l}\text { conversion factor } \\
\text { readout calib. }\end{array}$ & $\eta<2.4$ & $5 \times 10^{31}$ & chamber & $5 \mathrm{~min}$ & n.a. & n.a. & 0.01 \\
\hline $\begin{array}{l}\text { MDT } \\
\text { hits }\end{array}$ & $\begin{array}{l}\text { Efficiency, strip } \\
\text { noise, pileup }\end{array}$ & $\eta<2.4$ & $10^{31}$ & $\begin{array}{l}4 \times 10^{5} \\
\text { tubes }\end{array}$ & $\sim$ hours & $\begin{array}{c}\mathrm{n}, \gamma \\
\text { charged }\end{array}$ & $\begin{array}{l}600 \mathrm{~ns} \\
\mu \mathrm{s} / \mathrm{evt}\end{array}$ & 2.4 \\
\hline $\begin{array}{l}\text { TGC } \\
\text { hits }\end{array}$ & $\begin{array}{l}\text { Efficiency } \\
\text { strip noise }\end{array}$ & $\begin{array}{c}1.05<\eta \\
\eta<2.4\end{array}$ & $\begin{array}{c}\text { not } \\
\text { estimated }\end{array}$ & $\begin{array}{l}3 \times 10^{5} \\
\text { strips }\end{array}$ & $\sim$ hours & & $\sim 10 \mathrm{~ns}$ & $\begin{array}{c}35 \\
\text { ns/evt }\end{array}$ \\
\hline $\begin{array}{l}\text { CSC } \\
\text { hits }\end{array}$ & $\begin{array}{l}\text { Efficiency } \\
\text { strip noise }\end{array}$ & $\begin{array}{c}1.98<\eta \\
\eta<2.7\end{array}$ & $\begin{array}{c}\text { not } \\
\text { estimated }\end{array}$ & $\begin{array}{c}3 \times 10^{4} \\
2 \mathrm{D} \text { strips }\end{array}$ & $\sim$ hours & $\begin{array}{c}\mathrm{n}, \gamma \\
\text { charged }\end{array}$ & $\sim 10 \mathrm{~ns}$ & $\begin{array}{c}80 \\
\text { ns/evt }\end{array}$ \\
\hline
\end{tabular}

Table 1. Features of the CSC, MDT, RPC and TGC detector technologies, relevant for the background measurement

\subsection{Data Monte Carlo Comparison}

A FLUGG-based application simulates individual proton-proton collisions whose daughter particles are followed through the ATLAS detector materials. As they enter the muon detectors logical volumes they are scored and converted to particle fluxes according to the respective sensitivity functions which depend on the particle type and energy. More details about the FLUGG-based simulation can be found in [18].

The comparison of RPC data vs. FLUGG predictions is performed by converting the MC counts in each scoring volume to an equivalent rate at a given luminosity using the following:

$$
\mathrm{MC} \text { hit rate }\left[\mathrm{Hz} / \mathrm{cm}^{2}\right] R_{\mathrm{MC}}=\frac{n_{\mathrm{hit}, \mathrm{MC}}}{\varepsilon n_{\mathrm{events}, \mathrm{MC}}} \cdot \mathscr{L} \cdot \sigma_{\mathrm{pp}} \cdot \frac{1}{A}
$$

where $\mathscr{L}$ corresponds to the instantaneous luminosity at which the data have been sampled, $n_{\mathrm{hit}, \mathrm{MC}}$ the number of hits on a given surface $A, n_{\text {events,MC }}$ the total number of simulated events, $\varepsilon$ an 
enhancement factor for the detector sensitivity to improve the statistics, and $\sigma_{\mathrm{pp}}$ the proton-proton cross section.

In the present case, the LHC fill 2110 starting September the 15th 2011 has been taken as a reference and the data represent the average of three consecutive samples of $30 \mathrm{~s}$ each with an average luminosity of $2.94 \times 10^{33} \mathrm{~cm}^{-2} \mathrm{~s}^{-1}$. To improve the comparison significance and to overcome the statistics limitation of the present FLUGG sample the data and the Monte Carlo hits have been summed over respectively the coordinate $\phi$ and $z$ of the barrel, obtaining $z$ and $\phi$ projections. The comparison study is summarized in figures 11 and 12 where the $z$ projection of the odd and even sectors and the $\phi$ projection of all sectors are shown, respectively. The data are normalized to $\mathscr{L}=10^{33} \mathrm{~cm}^{-2} \mathrm{~s}^{-1}$. The data are further integrated on the two gas-gap layers of a chamber, and for the middle stations the Pivot and Confirm chambers have been summed together as done in the FLUGG simulation where they correspond to a single scoring volume. Due to minor differences between the scoring volumes on the MC simulation and the real chamber sizes, in some specific detector area the MC counts have been scaled linearly to fit the real chamber areas. Each projection is completed with an auxiliary plot showing per each bin the fractional difference between data and MC: $($ Data $-M C) /$ Data. Each figure shows from left to right the middle layer and the outer layer chambers results respectively.

As noted before there are localized excesses of MC counts with respect to the measured signal in correspondence of the shielding cracks at $z \sim 3 \mathrm{~m}$ and $z \sim 7 \mathrm{~m}$. Another systematic effect is visible on the outer layer chambers showing a significative lower counting in the central values of $z$ with respect to the MC. This effect is much reduced on the middle layer chambers and almost suppressed on the large chambers (BML). Looking at $\phi$ projection it is clear that the even sectors show an overall effect of lower counting with respect to the $\mathrm{MC}$ at all $z$ values, more pronounced in the outer layer chambers; this overall effect is systematically concentrated in the lowermost sectors.

An analysis of this discrepancies can be done by separating the scaling factor error which may be attributed to a conversion factor and/or to the sensitivity function hardwired in the MC. In both cases we must also consider a non uniform term due to the approximations concerning the heavy ionizing particles such as the neutrons, which have a non uniform distribution. This effect would show as an excess of counts where there is a higher concentration of neutron hits (typically at higher $\eta)$. A better knowledge of the neutron distribution and detector response function is needed to go beyond the present level of comprehension at this point. The systematic effect on the differences shown in the distributions of figures 11 and 12 , seems to be related to passive material which is more concentrated in the ATLAS barrel feet area.

\section{Luminosity and Detector Activation}

The RPC detector currents during normal running show a clear correlation with the LHC operation: this information can be used as a monitor to estimate the instantaneous radiation or to spot any problematic behavior related to bad gas supply or anomalous detector ageing. The correlation of the gap currents with the instantaneous luminosity has been studied aiming at an independent measurement of this quantity with the RPCs. Considering that the RPCs are few meters away from the beam pipe, the measurement should be insensitive to beam halo background and to temperature 

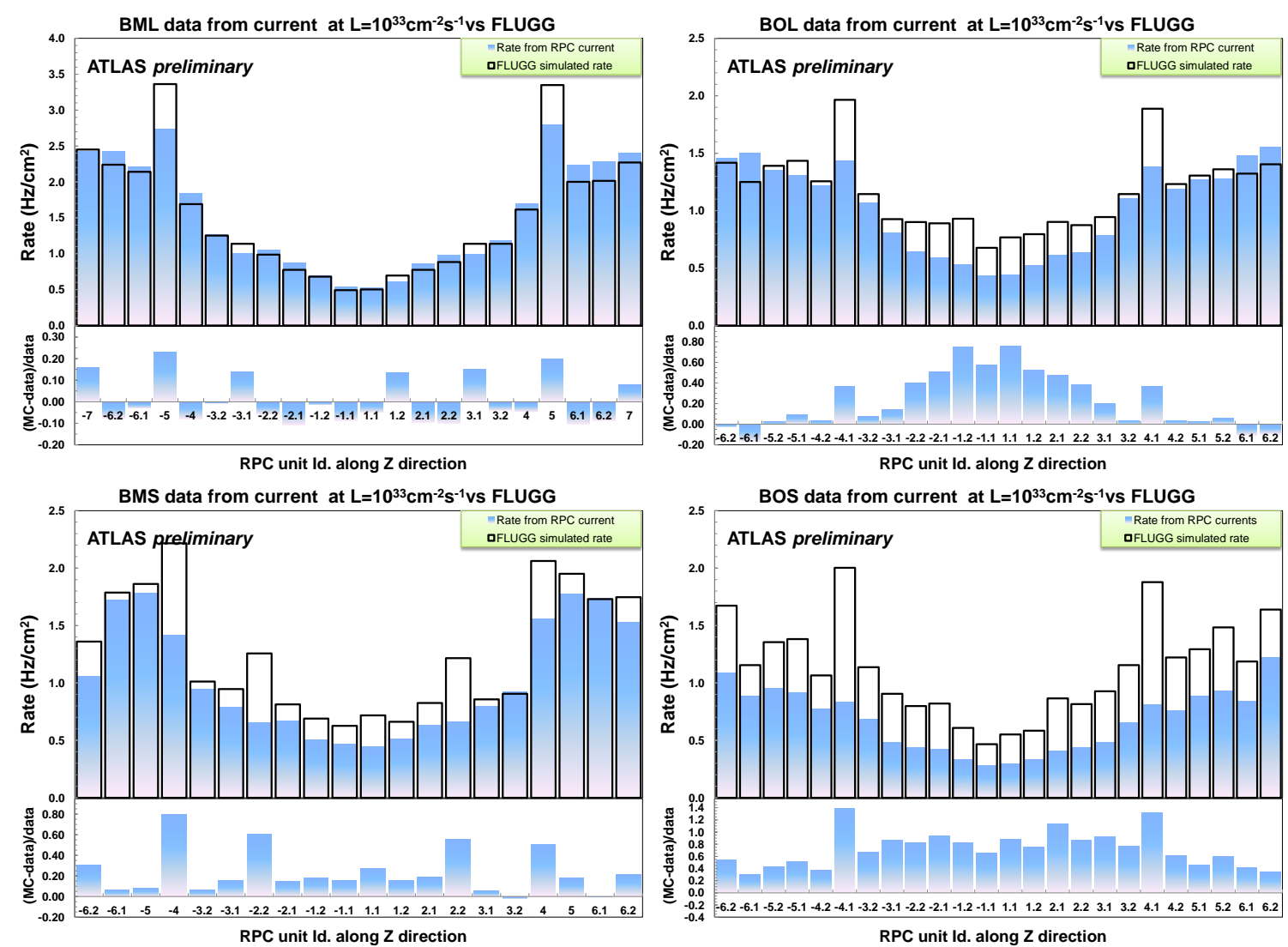

Figure 11. Data to FLUGG-based simulation comparison for large (even) and small (odd) sectors of the middle and outer layers. The data are integrated over the azimuthal coordinate $\phi$ and projected on the $z$ axis. Each bin of the top panel shows the rate in $\mathrm{Hz} / \mathrm{cm}^{2}$ averaged on all the gas-gaps with the same position in $z$, indicated by the chamber id.

and atmospheric pressure changes due to the the automatic adjustment of the high voltage working point.

The most straightforward quantity to estimate the luminosity can be obtained by combining all RPC gas-gap currents properly pedestal subtracted and normalized by their active surface:

$$
\Delta I_{R P C}=\sum_{j} \frac{I^{j}-I_{0}^{j}}{A^{j}}
$$

where $I^{j}$ are the gap currents, $I_{0}^{j}$ the current pedestals taken with no beam in the machine and at same voltage and $A^{j}$ are the active gap areas. Those few chambers showing a problematic trend due to detector, gas flow or electronics problems are manually flagged and removed from the computation.

For safe operation, the RPCs are automatically set at one of two High Voltage (HV) configurations depending on the LHC beam conditions. These are:

- the READY state, with the detector at full voltage/gain/efficiency $(\sim 9600 \mathrm{~V})$; the RPC are required to reach that configuration as soon as the LHC declares stable beams; 

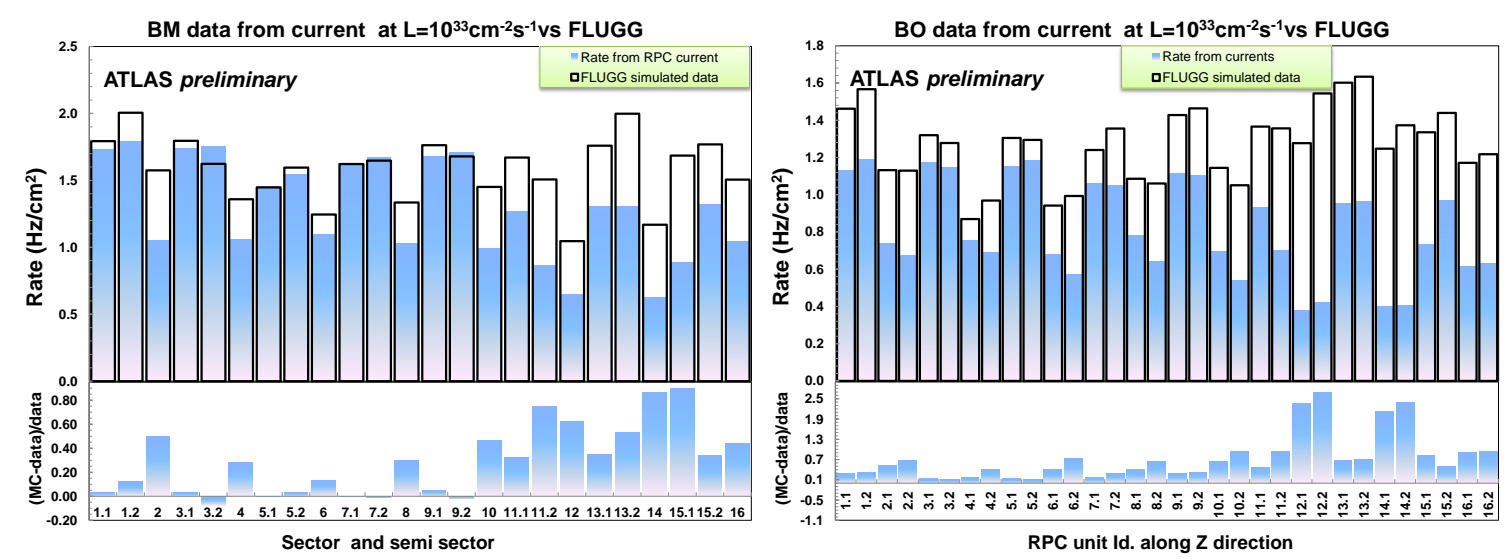

Figure 12. Data to FLUGG-based simulation comparison for the middle and outer layers. The data are integrated over the coordinate $z$ and projected on the $\phi$ axis. Each bin of the top panel shows the rate in $\mathrm{Hz} / \mathrm{cm}^{2}$ averaged on all the gas-gaps within the same position in $\phi$, generally corresponding to an extension of one half of a sector, except for the small barrel middle chambers (BMS) which havw only one gap covering the sector width.

- the STANDBY state, with the detector at lower voltage/gain/efficiency ( $\sim 9000 \mathrm{~V})$; this configuration is requested whenever the LHC is injecting, ramping or squeezing the beams.

In order to be less sensitive to changes in the detector settings or the LHC luminosity first measurements were performed for the following two well defined conditions:

- at the beginning of the fill, when the beams start colliding but before the stable beams declaration, in order to avoid the detector ramp up to READY state;

- at the end of the fill, when the beams are dumped and before the ramp down of the detector to STANDBY state.

The RPCs HV were at a fixed value during these beam transition phases which made it possible to estimate the contribution to the current due to the beam collisions. In the first case the detector was in STANDBY state; in the second case in READY state. The typical behaviour of the RPCs can be observed in by directly looking at a DCS plot (see Fig. 113 a), where the current follows the ATLAS instantaneous luminosity. A first positive step of the current can be seen at the beginning of the fill, when the detector was still at lower voltages $(9000 \mathrm{~V})$. At the end of the fill a negative step is also visible, occurring when the beams were dumped. Once the fill is over, a residual current (indicated as "Pedestal" in Fig. 13a) is present, corresponding to the detector dark current at $9600 \mathrm{~V}$. The observed current differences $\triangle I_{R P C}$ for the two conditions were then associated to a luminosity difference $\Delta \mathscr{L}$. Fig. $13 \mathrm{~b}$ b shows the instantaneous luminosity versus the RPCs current for the two voltage gains. The data, obtained in several fills, show a clear linear relation between current and luminosity. The slopes of the two curves are different due to the different detector gains.

\subsection{Measurement Tecnique and Results}

One of the main systematic effects is the strong influence of the environmental parameters on the detector behaviour. A dynamic adjustment of the high voltage working point as a function of the 

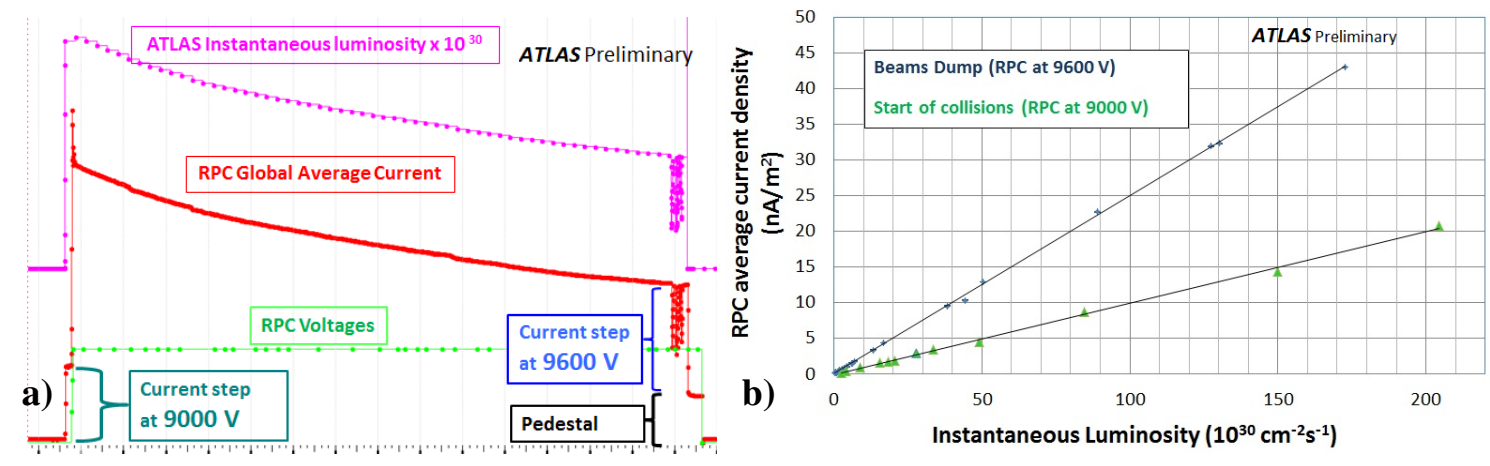

Figure 13. a) An example of the RPCs average current and the ATLAS instantaneous luminosity as a function of time before, during and after the LHC fill are shown. A pedestal is visible at the end of the fill, corresponding to the detector dark current at $9600 \mathrm{~V}$. b) The RPCs average current (per unit area and pedestal subtracted) versus the ATLAS instantaneous luminosity at the start of the collisions (green triangles) and at the beam dump (blue stars).

local environmental conditions (temperature and atmospheric pressure) was necessary for a stable operation. For technical reasons it was only possible to apply this correction when the detector was at full voltage $(9600 \mathrm{~V})$. In this case the RPCs current was expected to be strictly correlated to the beam collisions and not influenced by other factors. Consequently the analysis efforts have been concentrated on the extraction of the corrected values of current at beam dumps.

Fig. 114 shows a collection of several measurements of average current density (pedestal substracted) versus instantaneous luminosity at beam dump. The measurements refer to 2010 and 2011 data and span over a range of more than 4 decades. Data fits a straight line with a slope of 0.312 $\pm 0.001 \mathrm{nA} \mathrm{m} \mathrm{m}^{-2} / 10^{30} \mathrm{~cm}^{-2} \mathrm{~s}^{-1}$ and a negligible intercept. This result shows that the ratio between the ATLAS luminosity and the RPCs current $(\Delta I)$ is remarkably constant. Thus, demonstrating that an independent online luminosity measurement is possible once calibrated.

As a by product of this analysis, it was possible to extrapolate the fit to the LHC project luminosity of $\mathscr{L}=10^{34} \mathrm{~cm}^{-2} \mathrm{~s}^{-1}$. From the extracted value of the current, an average counting rate of $\sim 10 \mathrm{~Hz} / \mathrm{cm}^{2}$ was predicted, by using a $30 \mathrm{pC} /$ count conversion factor. This result together with several 2-dimensional maps of the current have been extensively used for ATLAS upgrade studies, in order to have a reliable estimation and description of the hit rate for higher LHC luminosity.

Looking in more detail at the current trends after beam dump, an interesting effect of ATLAS cavern activation has been observed. A very important effect that had to be understood, quantified and constantly monitored. This observation was possible even if the time needed by the DCS to update and calculate the average value of the currents was not optimal. Approximately every 30 seconds an independent data set is provided, thus implying a step in the RPCs current 30-40 seconds after a beam dump. Rather than dropping to the expected detector dark current $\left(\sim 100 \mathrm{nAm}^{-2}\right)$, the trends were instead described by an exponential decay function.

The lifetime $(\tau)$ and the intensity $\left(A_{0}\right)$ of the exponential decay function have been extracted, analysing data for several fills with different instantaneous luminosities at beam dump. In order to have a reasonable amount of data to fit, the detector was kept regularly at full voltages for 20 minutes after each dump. 


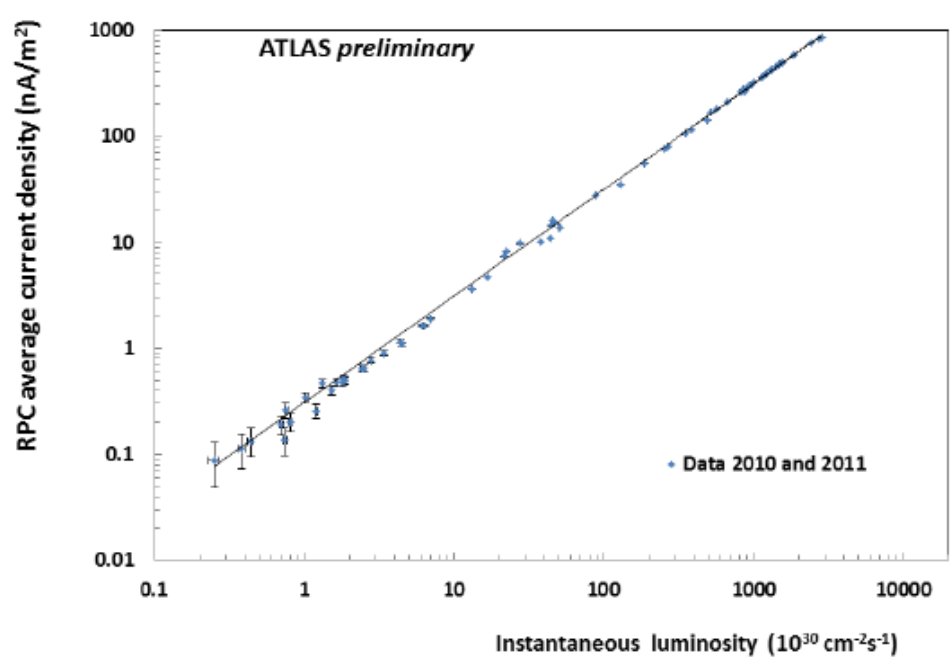

Figure 14. The RPC average current density (pedestal subtracted) as a function of the instantaneous luminosity at beam dump. The measurements include both 2010 and 2011 data and span over a range of more than four orders of magnitude. Data fit a straight line with a slope of $0.312 \pm 0.001 \mathrm{nA} \mathrm{m}-2 / 10^{30} \mathrm{~cm}^{-2} \mathrm{~s}^{-1}$ and a negligible intercept.

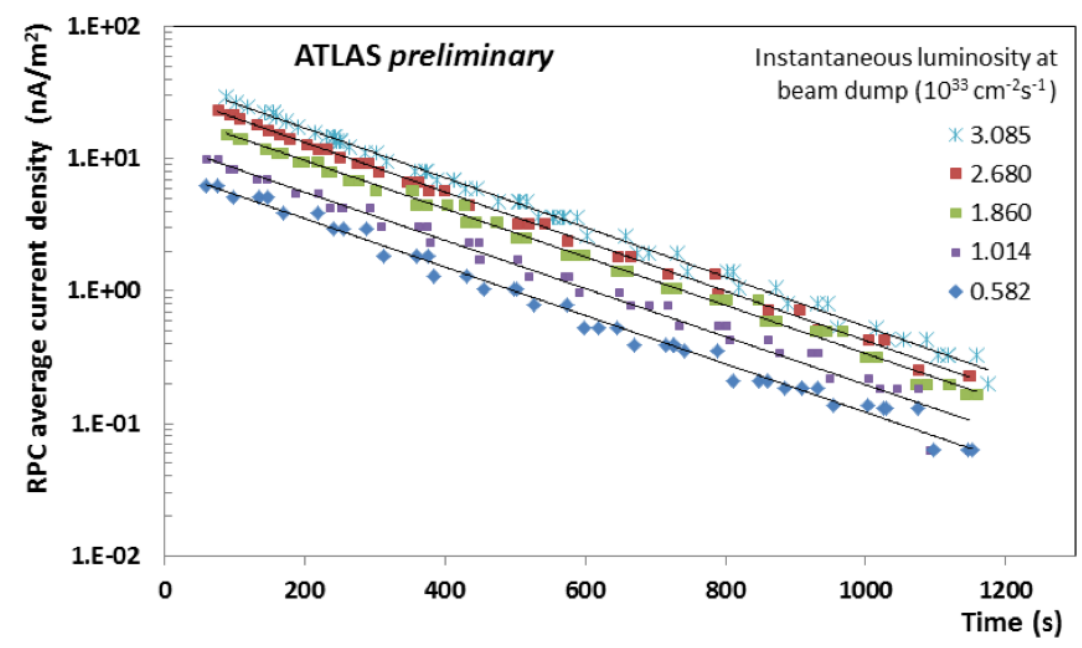

Figure 15. RPC average current density as a function of time after beam dump for different instantaneous luminosities.

Fig. 15 shows the results for a sub-sample of LHC fills where the RPCs current is shown as a function of time after beam dump for instantaneous luminosities ranging from 0.6 to 3.1 . $10^{33} \mathrm{~cm}^{-2} \mathrm{~s}^{-1}$. The results of the fits are summarised in Table 2. The decay rate is almost independent from the instantaneous luminosity and has an estimated average lifetime of $\langle\tau\rangle=(234 \pm 1)$ s. This result is similar to the one found by the other ATLAS muon detectors ( $200 \mathrm{~s})$. The materials inside the RPCs, interested by the activation, are mainly Aluminium and Copper but the relative contribution of each element to the decay constant is not trivial. The value should be compared 
with $\tau \simeq 404 \mathrm{~s}$ for the $C u^{64}$, and $\tau \simeq 208 \mathrm{~s}$ for the Aluminium isotope $\mathrm{Al}_{13}^{28}$, formed if a neutron is added to the stable isotope $\mathrm{Al}_{13}^{27}$.

In Fig. 16, the total current, the current induced by activation and their ratio (activation current/total current) are shown as a function of the instantaneous luminosity at beam dump. The activation current $\left(A_{0}\right)$ appears to depend linearly on the instantaneous luminosity. The ratio has been calculated and found to be almost constant with luminosity. The average value of the ratio is $(4.1 \pm 0.1) \%$.

\begin{tabular}{|c|c|c|c|c|}
\hline $\begin{array}{c}\text { Instantaneous Luminosity } \\
\left(\times 10^{33} \mathrm{~cm}^{-2} \mathrm{~s}^{-1}\right)\end{array}$ & $\mathbf{A}_{\mathbf{0}}\left(n A / \mathrm{m}^{2}\right)$ & $\boldsymbol{\delta} \mathbf{A}_{\mathbf{0}}\left(n A / \mathrm{m}^{2}\right)$ & $\tau(s)$ & $\delta \tau(s)$ \\
\hline 3.085 & 40 & 1 & 232 & 2 \\
\hline 2.680 & 31 & 1 & 232 & 2 \\
\hline 1.860 & 22 & 1 & 235 & 3 \\
\hline 1.014 & 13 & 1 & 234 & 5 \\
\hline 0.582 & 8 & 1 & 237 & 4 \\
\hline
\end{tabular}

Table 2. The distributions have been fit with an exponential decay function $\mathrm{y}=\mathrm{A}_{0} * \exp (-\mathrm{t} / \tau)$ with $<\tau>=(234 \pm 1) \mathrm{s}$

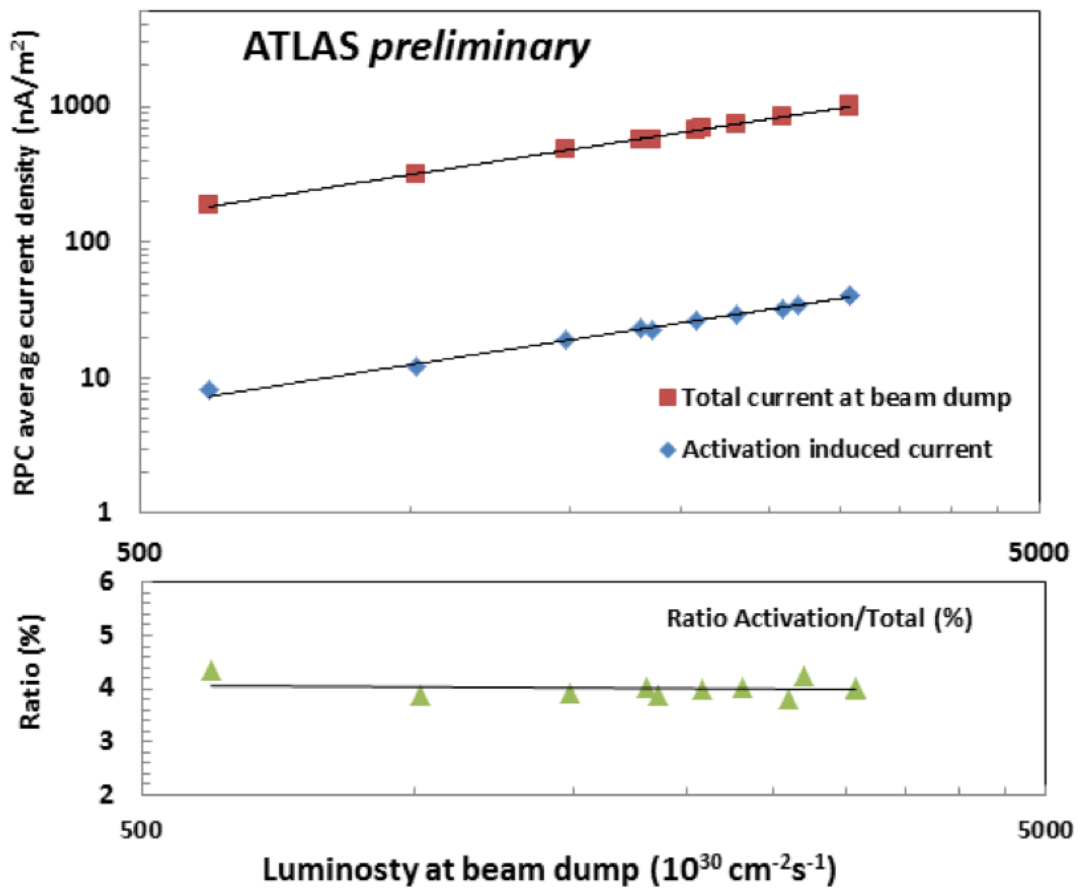

Figure 16. RPC Total average current, activation induced current (top) and their ratio activation/total current (bottom) as a function of the instantaneous luminosity at beam dump.

\subsection{Comparison with ATLAS Measurements and Future Prospects}

The ratio between the ATLAS luminosity and the current in the RPC is remarkably constant as 
shown in Fig. 14. Before providing an independent luminosity measurement some control checks were necessary.

As a first step, it was decided to monitor through the DCS the online ratio:

$$
R(t)=\frac{L_{A T L A S}(t)}{\Delta I_{R P C}(t)}
$$

in order to see if any systematic deviation was present during the LHC fill. No significant effects were observed. A further systematic check was the extraction of the ratio $\mathrm{R}(\mathrm{t})$ at the beam dump for several fills where the conditions of the RPCs detector or the LHC bunch configuration were different. Some examples of modified conditions are listed here:

- before and after the LHC technical stops, when major interventions on the detector occurred;

- a different gap selection (used by the averaging algorithm);

- a different LHC bunch configuration.

Fig. 17 shows a quantitative comparisons between the reference ratio $R\left(t_{0}\right)$, extracted from a calibration run (at beam dump) and the ratios calculated for several fills with different number of colliding bunches during the 2011 data taking. It can be noticed that a maximum deviation of $\pm 1.5 \%$ was achieved; for the other systematic checks, similar or smaller fluctuations have been observed. The agreement and the stability of the result can be considered satisfactory as can be seen also for a single luminosity fill in Fig. 18. In order to quote the RPCs luminosity for 2012, a new reference ratio $R\left(t_{1}\right)$ will be used to calibrate the current with the ATLAS online luminosity measurement. This is mainly due to the changes on ATLAS shielding and LHC beam energies respect to 2011. Once this ratio is fixed, it will be possible to provide an independent measurement based on the following formula:

$$
L_{R P C}=R\left(t_{1}\right) \cdot \Delta I_{R P C}
$$

During the whole 2012, RPCs will be providing ATLAS with an independent luminosity measurement that will be used to monitor and understand potential discrepancies. The fact that the activation current was found to scale linearly with the luminosity, means that it will not be necessary to add a further systematic to the measurement.

\section{Conclusions}

The ATLAS RPC have worked very well in 2011 delivering good trigger and data for physics. The detector redundancies along with the extensive large monitoring capabilities allowed to quantify the linear correlation between the LHC luminosity and the RPCs average current over four order of magnitude. A precise estimation of the cavern activation has been extracted and found to be constant as a function of the luminosity. A map of the cavern background as measured by the RPC has been extracted and compared to the available Monte Carlo description. The discrepancies found have allowed a better estimation and improvement of the available simulation. The encouraging results obtained will be verified during 2012 data taking, where RPCs will be measuring the online luminosity together with the other ATLAS measurements. The running of an extended system as the ATLAS RPC has provided a unique opportunity to improve the understanding of the physics 


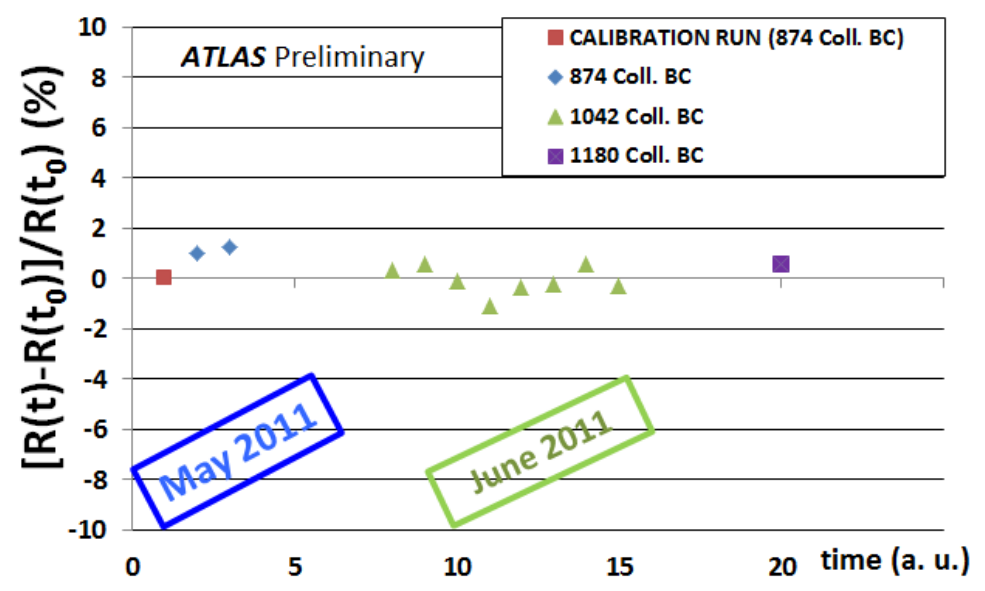

Figure 17. Percentage differences between the ratio $R(t)$ and the reference $R\left(t_{0}\right)$ (red square) calculated at beam dump for fills with a different number of colliding bunches.

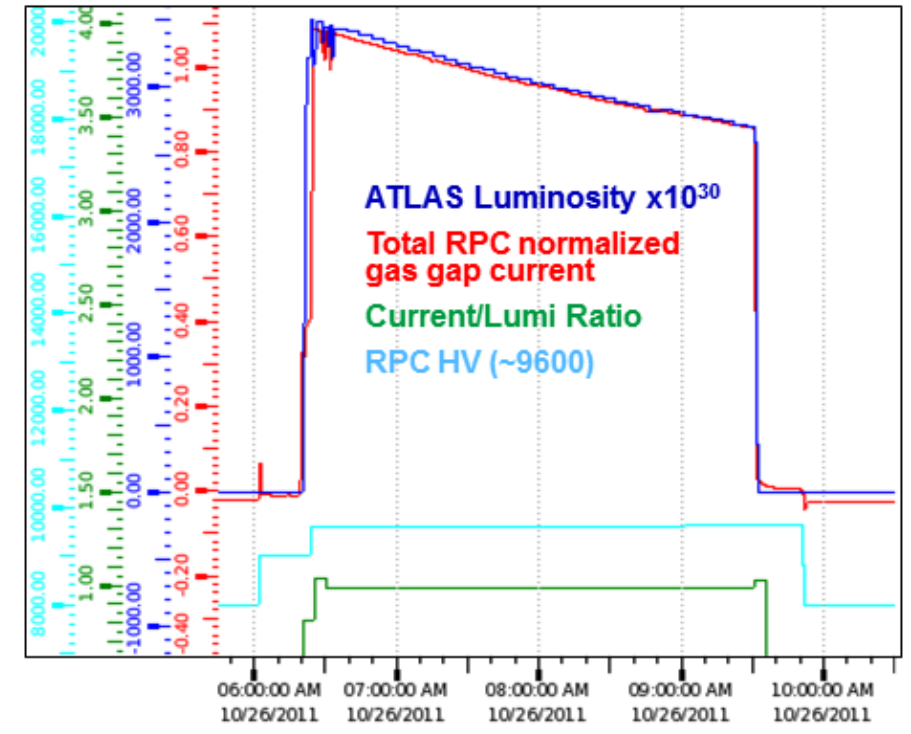

Figure 18. ATLAS and RPC Online instantaneous luminosity. The ratio between the two quantities is remarkably constant allowing, once calibrated, for an independent online luminosity measurement and studies on cavern activation background at beam dump.

of the detectors beyond that obtained from previous studies of aging and performance generally limited by the small number of detectors under test. The experience gained in the detector operation will help to plan the data taking for the next years and the system upgrades in view of the future high luminosity runs at the LHC.

\section{References}

[1] R. Santonico R. Cardarelli: "Development of resistive plate counters", NIM A, 187 (1981) 377. 
[2] ATLAS Muon Collaboration, "ATLAS Muon Spectrometer Technical Design Report", CERN/LHCC/97-22, (1997)

[3] F. Anulli et al., "The Level-1 Trigger Muon Barrel System of the ATLAS experiment at CERN", 2009 JINST 4 P04010

[4] A.Polini, "Performance and Operation of the ATLAS Resistive Plate Chambers Detector in 2011", RPC 2012, Frascati 2012, being published.

[5] G. Chiodini, "The ATLAS RPC time-of-flight performance", RPC 2012, Frascati 2012, being published.

[6] A. Polini, "Design and Performance of the Detector Control System of the ATLAS Resistive-Plate-Chamber Muon Spectrometer", doi:10.1016/j.nima.2010.08.006, Nucl. Instrum. Meth. A661 (2012) 15-18

[7] B. Bilkid et al., "Environmental Dependence of the Performance of Resistive Plate Chambers", NIM: Nucl.Instrum.Meth.A 602 (2009) 700-704

[8] M. Bindi, "Operation and performance tuning of ATLAS RPCs through the detector control system at the startup of 2009 LHC run”, doi:10.1016/j.nima.2010.09.124, Nucl. Instrum. Meth. A661 (2012) $10-14$

[9] E. Pastori, “The ATLAS RPC Gas Distribution”, RPC 2012, Frascati 2012, being published.

[10] G. Aielli et al.; "The RPC current time structure. Fast current peak measurement in the ATLAS RPC system”, Nucl. Instr. and Meth. A 661 (2012) 201-205.

[11] G. Aielli et al.; "RPCs ageing studies"; Proc. of VCI 2001, Nucl. Instr. and Meth. A 478 (2002) 271-276

[12] G. Aielli et al.; “An RPC $\gamma$ irradiation test”, Nucl. Instr. and Meth. A 414 (1998) 82-86.

[13] S. Baranov et al.; "Estimation of Radiation Background, Impact on Detectors, Activation and Shielding", ATL-GEN-2005-001

[14] G. Aielli et al.; "Saturated logistic avalanche model", Nucl. Instr. and Meth. A 508 (2003) 6-13

[15] M. Bindi, "ATLAS RPC detector as Luminosity monitor", RPC 2012, Frascati 2012, these proceedings

[16] G. Aielli, "Cavern background measurement with the ATLAS RPC system", RPC 2012, Frascati 2012, these proceedings

[17] The ATLAS Collaboration; Cavern background measurements and comparison with simulation; 2012; ATLAS note in preparation

[18] FLUGG, http://www.fluka.org/content/tools/flugg/index.html 